\title{
Maternal tobacco, cannabis and alcohol use during pregnancy and risk of adolescent psychotic symptoms in offspring
}

\author{
Stanley Zammit, Kate Thomas, Andrew Thompson, Jeremy Horwood, Paulo Menezes, \\ David Gunnell, Chris Hollis, Dieter Wolke, Glyn Lewis and Glynn Harrison
}

\section{Background}

Adverse effects of maternal substance use during pregnancy on fetal development may increase risk of psychopathology.

\section{Aims \\ To examine whether maternal use of tobacco, cannabis or alcohol during pregnancy increases risk of offspring psychotic symptoms.}

\section{Method}

A longitudinal study of 6356 adolescents, age 12, who completed a semi-structured interview for psychotic symptoms in the Avon Longitudinal Study of Parents and Children (ALSPAC) birth cohort.

\section{Results}

Frequency of maternal tobacco use during pregnancy was associated with increased risk of suspected or definite psychotic symptoms (adjusted odds ratio 1.20, 95\% Cl 1.051.37, $P=0.007$ ). Maternal alcohol use showed a non-linear association with psychotic symptoms, with this effect almost exclusively in the offspring of women drinking $>21$ units weekly. Maternal cannabis use was not associated with psychotic symptoms. Results for paternal smoking during pregnancy and maternal smoking post-pregnancy lend some support for a causal effect of tobacco exposure in utero on development of psychotic experiences.

\section{Conclusions}

These findings indicate that risk factors for development of non-clinical psychotic experiences may operate during early development. Future studies of how in utero exposure to tobacco affects cerebral development and function may lead to increased understanding of the pathogenesis of psychotic phenomena.

\section{Declaration of interest}

None.
In the UK, $15-20 \%$ of women continue to smoke throughout their pregnancy, ${ }^{1}$ and although cannabis use is less common, some alcohol intake during pregnancy is reported by most women. ${ }^{2}$ Maternal substance use during pregnancy poses a potential risk to the health of the developing fetus, as tobacco, alcohol and cannabis all cross the placenta and the fetal blood-brain barrier. It has become increasingly clear within the field of teratology that exogenous agents that are relatively harmless to the mother during pregnancy may have detrimental neurological effects on the child, and that most such effects are subtle and may not be detectable at birth. $^{3,4}$

There is robust evidence from epidemiological and animal model studies that maternal tobacco use during pregnancy leads to a number of adverse perinatal outcomes. ${ }^{5-7}$ Maternal smoking has also been repeatedly reported as being associated with adverse long-term effects on the offspring, including reduced cognitive ability ${ }^{7-9}$ and increased incidence of attention-deficit hyperactivity disorder and conduct disorder during childhood and adolescence, ${ }^{6,7,10-12}$ with similar, though less consistent, reports for maternal cannabis and alcohol use. ${ }^{4,8}$

It is difficult to be confident that any associations between maternal substance use and child psychopathology from epidemiological studies are causal and not confounded by other characteristics of women who continue to use substances during pregnancy. Methods that would help causal inference include comparing the associations observed for maternal and paternal substance use during pregnancy. ${ }^{13}$ However, very few studies of childhood psychopathology have utilised such approaches to date.

Animal models of psychopathology are more difficult to examine than models of perinatal morbidity such as birth weight.
Nevertheless, animal studies show that exposure to nicotine in utero, ${ }^{14,15}$ and to a lesser extent exposure to alcohol and cannabinoids, ${ }^{4,16}$ can lead to profound and lasting changes in cerebral development and neurotransmitter function, making maternal substance use in pregnancy a very plausible risk factor for offspring psychopathology.

Given that a wide range of obstetric and pregnancy complications have been associated with risk of schizophrenia in offspring, ${ }^{17}$ it is surprising that there are so few studies that have focused on the effects of maternal substance use on risk of psychosis. In the systematic review by Cannon et al, ${ }^{17}$ there were only two studies identified on maternal smoking. ${ }^{18,19}$ There was no evidence of association between maternal smoking and schizophrenia in that meta-analysis, ${ }^{17}$ but the statistical power is likely to have been relatively low. More recently, maternal smoking in pregnancy was associated with the presence of psychotic symptoms in the Early Developmental Stages of Psychopathology (EDSP) study of almost 1000 young adults in Munich. ${ }^{20}$ However, as this was a cross-sectional design with retrospective recall of maternal behaviour during pregnancy, recall bias is difficult to exclude.

Our aim was to investigate, in a longitudinal design, whether maternal tobacco, cannabis or alcohol use during pregnancy were independently associated with risk of the offspring developing psychotic symptoms during early adolescence. We hypothesised that maternal tobacco, and to a lesser extent cannabis and alcohol use, would be associated with psychotic symptoms, and that a substantial part of this association would be mediated through effects of maternal substance use on adverse perinatal outcomes and cognitive ability during childhood. 


\section{Method}

\section{Sample}

This study examined data from 6356 children from the Avon Longitudinal Study of Parents and Children (ALSPAC) cohort who participated in the psychosis-like symptoms (PLIKS) semistructured interview (PLIKSi) ${ }^{21}$ when they were 12 years of age (data restricted to one child per nuclear family). The initial cohort consisted of 14062 children born to residents of the former Avon Health Authority area who had an expected date of delivery between 1 April 1991 and 31 December 1992 (www.alspac.bris.ac.uk). The cohort was set up to examine genetic and environmental determinants of health (including mental health) and development. ${ }^{22}$ The parents have completed regular postal questionnaires concerning their child's health and development since birth. The children, since 7.5 years of age, have attended annual assessment clinics where they participate in a range of face-to-face interviews. Due to wave non-response, sample sizes in the analyses differ according to exposures and data-sets examined (see Results and Tables). Non-attendees participating in the PLIKSi were more likely to come from lower social class families, to have parents with lower education, to be male and to be of minority ethnic status. ${ }^{21}$

\section{Measures}

\section{Outcomes}

The PLIKSi consists of 12 core questions covering hallucinations (visual and auditory); delusions (delusions of being spied on, persecution, thoughts being read, reference, control, grandiose ability and other unspecified delusions); and experiences of thought interference (thought broadcasting, insertion and withdrawal) over the past 6 months. For these 12 core items, 7 screening (stem) questions were derived from DISC-IV ${ }^{23}$ and 5 questions from the Schedules for Clinical Assessment in Neuropsychiatry (SCAN) version 2.0, ${ }^{24}$ modified slightly after piloting (further details available at www.bristol.ac.uk/psychiatry/ staff/zammit/documents/pliks.pdf.html). Clinical cross-questioning and probing was used to establish the presence of symptoms, and coding of all items followed the glossary definitions and rating rules for SCAN. Interviewers (psychologists trained in using the PLIKSi) rated symptoms as either not present, suspected or definitely present. Unclear responses after probing were always 'rated down', and symptoms only rated as definite when a credible example was provided. We included symptoms in our analyses only if they were not attributable to effects of sleep, fever or substance use, consistent with the approach of classification systems for diagnosis of functional psychotic disorders. The average kappa value for interrater reliability was $0.72 .^{21}$

We examined two primary PLIKS outcomes: presence or absence of any suspected or definite symptoms; and a narrower outcome of definite symptoms only. As secondary analyses, we also examined whether associations were stronger for more frequently occurring symptoms (definite symptoms occurring monthly or more frequently), or for symptoms that were more characteristic of schizophrenia (any suspected or definite 'bizarre' PLIKS). These symptoms, in concordance with both DSM-IV ${ }^{25}$ and ICD $-10^{26}$ criteria for schizophrenia, included either thirdperson auditory hallucinations or first-rank delusions (delusions of control or delusions of thought broadcast, insertion or control).

\section{Exposures}

Data on parental substance use were obtained from self-report postal questionnaires completed by the mother at 8,18 and 32 weeks of pregnancy and at 2, 21, 33 and 47 months after birth of the child, and from questionnaires completed by the father at 18 weeks of pregnancy and at 2, 8 and 21 months after birth of the child. Maternal tobacco, cannabis and alcohol use were coded as the highest category of use during any trimester of pregnancy (tobacco: $0,1-9,10-19, \geqslant 20$ cigarettes per day; cannabis: 0, $<$ weekly, $\geqslant$ weekly use; alcohol: $0, \leqslant 7,8-21, \geqslant 22$ units per week). For alcohol we also used a continuous measure of units of alcohol per week during pregnancy (maximum of first and third trimester intake), divided by 10 to create estimates per 10unit increases in alcohol intake. We also examined whether effects for each substance were different according to trimester of exposure.

\section{Confounders}

A number of sociodemographic variables were considered as potential confounders: gender, parental social class (highest of both parents, based on occupation using the 1991 Office for Population Censuses and Surveys (OPCS) classification; ${ }^{27}$ coded as I-V (lowest)); maternal marital status during pregnancy (married, partner, single); financial difficulty during pregnancy; housing type (mortgaged/owned, privately rented, council); urban/rural index at birth (urban/town, village/hamlet); paternal smoking during pregnancy; and maternal and paternal education (four-levels, ranging from the lowest UK school-leaving qualifications up to degree level). We also considered parental age, maternal use of prescribed medication (analgesics or hypnotics), maternal depression during pregnancy (Edinburgh Postnatal Depression Scale $)^{28}$ and other family history of depression, schizophrenia or any mental health illness (in biological parents or grandparents) as potential confounders.

To further examine possible confounding we compared maternal smoking during pregnancy with paternal smoking during pregnancy and also with maternal smoking 2-3 years post-pregnancy, before and after adjusting for confounding and for the other two parental smoking variables. For alcohol use we further examined possible effects of confounding by examining the relationship between maternal alcohol use 4 years postpregnancy and PLIKS independently of effects of alcohol use during pregnancy.

Variables that we considered potential mediators of the relationship between maternal substance use during pregnancy and risk of PLIKS in the offspring (i.e. lying on the causal pathway) were child Wechsler Intelligence Scale for Children-III (WISC-III) total IQ score ${ }^{29}$ from assessment clinic at age 8 , and 5-minute Apgar score, gestation, and birth weight (as a marker of chronic effects on fetal growth), obtained from pregnancy records.

\section{Ethical approval}

Ethical approval for the study was obtained from the ALSPAC Law and Ethics Committee and the local research ethics committees.

\section{Statistical analysis}

Logistic regression was used to calculate odds ratios (ORs) and 95\% confidence intervals for associations between maternal substance use and PLIKS outcomes, both before and after adjustment for potential confounders. Our primary analyses for ordered categorical data (maternal tobacco and cannabis use) was to study a linear trend effect across increasing categories of use. In order to investigate a non-linear relationship for alcohol use, a quadratic term was used in addition to a linear term, and likelihood ratios tests (LRT) used to examine evidence of a 
non-linear relationship; and overall effect of alcohol use (linear and quadratic) on PLIKS outcomes.

\section{Missing data}

Children not attending the PLIKSi were more likely to have mothers who smoked $(35.1 \%)$ and used cannabis $(3.6 \%)$ compared with those who did attend $(19.4 \%$ and $2.6 \%, P<0.001$ for both). However, maternal alcohol use ( $>1$ glass per week) was less common in non-attendees $(21.8 \%$ v. $25.2 \%, P<0.001)$. To examine whether missing data may have biased our results we conducted sensitivity analyses using multiple imputations by chained equations. ${ }^{30,31}$ We used the ice command in Stata for Windows (version 9) to impute missing data for confounders and outcomes. Fifty variables relating to parental sociodemographic, and child emotional, social and behavioural characteristics were used to impute missing data. Ten cycles of regression were carried out and 25 data-sets imputed.

\section{Results}

There were 734 children $(11.6 \%$ of those interviewed, $95 \%$ CI $10.8-12.4 \%$ ) who were rated as having suspected or definite PILKS, and 300 of these (4.7\% of those interviewed) had definite symptoms. A summary of the potential confounders in relation to maternal substance use is presented in Table 1. Individually adjusting for gender, other family history of mental health illness and paternal age made minimal difference to any of the results and these were therefore omitted from the analyses.

Of the children interviewed for PLIKS, there were 6332 with maternal smoking data, 6210 with maternal cannabis use data, and 6245 with maternal alcohol data available. Of these, 1219 $(19.3 \%)$ of mothers smoked tobacco, $4372(70.0 \%)$ of mothers drank alcohol, and 157 (2.5\%) of mothers took cannabis at least once during their pregnancy. There were 4253 adolescents with data available on PLIKS, confounders, and maternal use of tobacco, cannabis and alcohol, and this was the sample used for the main analyses.

\section{Tobacco use during pregnancy}

Maternal tobacco use during pregnancy was strongly associated with any suspected or definite PLIKS in the offspring (crude OR for linear trend across four smoking categories 1.33, 95\% CI 1.18-1.49), and results were consistent with a dose-response effect (Table 2). This was attenuated only partially after adjusting for confounders (adjusted OR $=1.20,95 \%$ CI 1.05-1.37, $P=0.007$ ). The two confounders that had the greatest effect on attenuating this estimate were paternal smoking and single status of the mother. Further adjusting for gestation, birth weight, 5-minute Apgar score, or age 8 IQ score, as possible mediators for this association, had minimal effects on these results. These estimates were similar for definite PLIKS as an outcome although results were less precise.

We further examined possible effects of confounding by studying the effects of paternal smoking during pregnancy and maternal smoking post-pregnancy on risk of PLIKS, to compare these with the effect of maternal smoking during pregnancy (Table 3). Paternal smoking during pregnancy was associated with any suspected or definite PLIKS in the crude analysis, but this was eliminated after adjusting for confounders and maternal smoking (adjusted OR $=1.05,95 \%$ CI $0.95-1.17$ ).

Maternal smoking post-pregnancy was also associated with any suspected or definite PLIKS in the crude analysis (Table 3), but again this was eliminated after adjusting for confounders and maternal smoking during pregnancy (adjusted $\mathrm{OR}=0.95$, 95\% CI 0.79-1.14). Maternal smoking during pregnancy and maternal smoking post-pregnancy were quite strongly correlated (Kendall's $\tau_{\mathrm{b}}=0.76$ ). The standard error for maternal smoking during pregnancy was increased by about $60 \%$ when both were included in the same model (Table 3), but that for maternal smoking post-pregnancy was relatively unchanged, indicating that collinearity is unlikely to explain the lack of association for smoking post-pregnancy. ${ }^{32}$ Note that only 3730 of the 4253 adolescents had additional data on maternal smoking postpregnancy and therefore results for maternal smoking during pregnancy in Tables 2 and 3 are slightly different as they are based on different data-sets.

We examined whether the effect of maternal tobacco use differed by trimester of exposure. Smoking during any trimester was very correlated with smoking in other trimesters (Kendall's $\tau_{\mathrm{b}}>0.80$ ). The offspring of mothers who used tobacco only in their third trimester had a greater risk of developing any suspected or definite PLIKS than offspring whose mothers smoked only in the first trimester (OR for smoking in third trimester only compared with first trimester only 2.1 , 95\% CI $0.96-4.59$, $P=0.063)$. There were insufficient numbers of women who only used tobacco in their second trimester to examine specific second trimester effects.

\section{Cannabis use during pregnancy}

Maternal cannabis use was not associated with any suspected or definite PLIKS in the crude analysis (OR for linear trend 1.22, 95\% CI 0.83-1.79). The odds ratio was reduced after adjusting

Table 1 Descriptive summary of confounders in relation to maternal substance use during pregnancy ${ }^{2}$

\begin{tabular}{|c|c|c|c|c|c|c|c|c|c|c|c|}
\hline & \multicolumn{11}{|c|}{$n(\%)$} \\
\hline & $\begin{array}{l}\text { Low } \\
\text { social } \\
\text { class }\end{array}$ & $\begin{array}{l}\text { Income } \\
\text { support }\end{array}$ & $\begin{array}{l}\text { Council } \\
\text { housing }\end{array}$ & $\begin{array}{l}\text { Single } \\
\text { status } \\
\text { (mother) }\end{array}$ & $\begin{array}{l}\text { Rural } \\
\text { birth }\end{array}$ & $\begin{array}{l}\text { Low } \\
\text { maternal } \\
\text { education }\end{array}$ & $\begin{array}{c}\text { Low } \\
\text { paternal } \\
\text { education }\end{array}$ & $\begin{array}{l}\text { Mother's } \\
\text { age >30 }\end{array}$ & $\begin{array}{l}\text { Medication } \\
\text { in } \\
\text { pregnancy }\end{array}$ & $\begin{array}{l}\text { Maternal } \\
\text { depression } \\
(\text { EPDS } \geqslant 15)\end{array}$ & $\begin{array}{c}\text { Paternal } \\
\text { tobacco } \\
\text { use }\end{array}$ \\
\hline \multicolumn{12}{|l|}{ Tobacco use } \\
\hline Non-smokers & $3462(39.9)$ & 561 (6.3) & 7787 (81.6) & 749 (7.7) & $623(6.3)$ & $1408(15.3)$ & $1328(17.9)$ & $3371(33.7)$ & $5882(62.2)$ & $453 \quad(4.8)$ & $1890(23.6)$ \\
\hline Smokers & $1722(60.2)$ & 585 (18.9) & 1815 (51.2) & $734(19.8)$ & 139 (3.7) & $1101(13.9)$ & 808 (33.9) & $803(20.9)$ & $2557(73.1)$ & $413(11.6)$ & $1829(68.6)$ \\
\hline \multicolumn{12}{|l|}{ Cannabis use } \\
\hline Never used & 4849 (44.3) & $1000(8.8)$ & $9153(75.3)$ & $1296(10.4)$ & $723(5.8)$ & $2255(19.3)$ & $1967(21.1)$ & $3913(30.8)$ & $7894(64.7)$ & $740 \quad(6.1)$ & 3348 (33.9) \\
\hline Ever used & $162(53.8)$ & $102(30.3)$ & $152(39.7)$ & $105(26.8)$ & $8(2.0)$ & $84(24.0)$ & $76(29.7)$ & 87 (21.4) & $272(71.0)$ & 64 (16.9) & $214(73.8)$ \\
\hline \multicolumn{12}{|l|}{ Alcohol use } \\
\hline$<1$ glass/week & $4052(47.2)$ & $864(9.6)$ & 7098 (73.2) & $1058(10.6)$ & $535(5.3)$ & $1931(20.9)$ & $1713(23.1)$ & $2756(27.0)$ & $6164(63.6)$ & $627 \quad(6.4)$ & 2809 (34.6) \\
\hline$\geqslant 1$ glass/week & $1000(36.7)$ & $254(9.1)$ & 2279 (76.3) & $376(12.3)$ & $212(6.9)$ & $490(16.8)$ & $381(16.9)$ & $1308(42.3)$ & $2093(69.4)$ & 199 (6.6) & $852(35.1)$ \\
\hline $\begin{array}{l}\text { EPDS, Edinburgh Postr } \\
\text { a. For tobacco use, all } \\
(P=0.70) \text {. Note that co }\end{array}$ & $\begin{array}{l}\text { Depression } \\
0.001 \text {; for car } \\
\text { unding variab }\end{array}$ & Scale. & d only for & irpose of & ole ar & for ar & ata in tab & on & (1) - - & $t+$ & LIKS dat \\
\hline
\end{tabular}




\begin{tabular}{|c|c|c|c|c|c|}
\hline & $n$ & $\begin{array}{l}\text { Suspected or definite PLIKS } \\
\text { Crude OR }(95 \% \mathrm{Cl})\end{array}$ & $\begin{array}{l}\text { Suspected or definite PLIKS } \\
\text { Adjusted OR }(95 \% \mathrm{Cl})\end{array}$ & $\begin{array}{c}\text { Definite PLIKS } \\
\text { Crude OR }(95 \% \mathrm{Cl})\end{array}$ & $\begin{array}{c}\text { Definite PLIKS } \\
\text { Adjusted OR }(95 \% \mathrm{CI})\end{array}$ \\
\hline \multicolumn{6}{|l|}{ Tobacco } \\
\hline None & 3579 & 1 & 1 & 1 & 1 \\
\hline 1-9 cigarettes/day & 295 & $1.15(0.79-1.66)$ & $0.92(0.63-1.36)$ & $1.53(0.92-2.53)$ & $1.25(0.73-2.14)$ \\
\hline 10-19 cigarettes/day & 266 & $1.88(1.35-2.61)$ & $1.47(1.02-2.12)$ & $2.33(1.48-3.66)$ & $1.65(0.99-2.75)$ \\
\hline$\geqslant 20$ cigarettes/day & 113 & $2.30(1.45-3.65)$ & $1.84(1.12-3.03)$ & $2.03(1.01-4.10)$ & $1.54(0.73-3.25)$ \\
\hline Linear trend & 4253 & $1.33(1.18-1.49), P<0.001$ & $1.20(1.05-1.37), P=0.007$ & $1.39(1.18-1.63), P<0.001$ & $1.21(1.01-1.47), P=0.047$ \\
\hline \multicolumn{6}{|l|}{ Cannabis } \\
\hline None & 4175 & 1 & 1 & 1 & 1 \\
\hline$<1 /$ week & 37 & $0.95(0.34-2.70)$ & $0.58(0.20-1.70)$ & $0.57(0.08-4.20)$ & $0.34(0.04-2.56)$ \\
\hline$\geqslant 1 /$ week & 41 & $1.62(0.71-3.66)$ & $1.04(0.45-2.43)$ & $1.63(0.50-5.32)$ & $1.12(0.33-3.84)$ \\
\hline Linear trend & 4253 & $1.22(0.83-1.79), P=0.317$ & $0.94(0.62-1.41), P=0.755$ & $1.16(0.65-2.09), P=0.616$ & $0.91(0.49-1.71), P=0.776$ \\
\hline \multicolumn{6}{|l|}{ Alcohol } \\
\hline None & 2522 & 1 & 1 & 1 & 1 \\
\hline$\leqslant 7$ units/week & 1293 & $0.92(0.74-1.14)$ & $0.92(0.74-1.15)$ & $0.67(0.48-0.94)$ & $0.68(0.48-0.96)$ \\
\hline 8-21 units/week & 410 & $1.05(0.77-1.48)$ & $1.00(0.71-1.39)$ & $0.58(0.33-1.04)$ & $0.56(0.31-1.02)$ \\
\hline$\geqslant 22$ units/week & 28 & $2.58(1.09-6.11)$ & $2.40(0.99-5.83)$ & $2.14(0.64-7.17)$ & $1.86(0.54-6.42)$ \\
\hline Linear (per 10 units) $^{\mathrm{b}}$ & 4253 & $0.80(0.51-1.25)$ & $0.75(0.47-1.19)$ & $0.77(0.48-1.25)$ & $0.73(0.45-1.18)$ \\
\hline Quadratic (linear $\left.{ }^{2}\right)^{\mathrm{b}}$ & 4253 & $1.21(1.00-1.47)$ & $1.22(1.00-1.49)$ & $1.04(0.97-1.12)$ & $1.04(0.97-1.12)$ \\
\hline $\begin{array}{l}\text { Likelihood ratio } \\
\text { (for overall alcohol) }^{\text {b }}\end{array}$ & 4253 & $\chi^{2}=9.8$, d.f. $=2, P=0.008$ & $\chi^{2}=8.3$, d.f. $=2, P=0.016$ & $\chi^{2}=1.3$, d.f. $=2, P=0.522$ & $\chi^{2}=1.8$, d.f. $=2, P=0.415$ \\
\hline
\end{tabular}

for confounders (Table 2), with adjustment for maternal tobacco use having the greatest impact on attenuation of this estimate (adjusted OR $=0.94,95 \%$ CI $0.62-1.41, P=0.755$ ). Of the 157 women with PLIKS data who used cannabis during pregnancy, $51(32.5 \%)$ claimed not to have smoked tobacco during their pregnancy. There were insufficient numbers of women using cannabis to examine trimester-specific effects of cannabis use.

\section{Alcohol use during pregnancy}

Although 70\% of mothers drank alcohol at least once during their pregnancy, the median number of units of alcohol per week consumed was 0 (range 0 to 102). There was an association between maternal alcohol intake during pregnancy and any suspected or definite PLIKS in the crude analysis (OR per 10-unit increase in alcohol 1.24, 95\% CI 1.03-1.50), and this was not substantially altered after adjustment (adjusted OR per 10 units 1.19, 95\% CI 0.97-1.45). This was a non-linear effect (likelihood ratio for inclusion of quadratic term in crude model $\chi^{2}=7.5$, d.f. $=1, P=0.006)$. Likelihood ratio test results for the overall effect of alcohol on risk of PLIKS are presented in Table 2. Further adjusting for possible mediators of this association had minimal effects on these results.
The increase in risk of suspected or definite PLIKS was primarily present in the offspring of the 25 mothers $(0.6 \%$ of the sample) who drank $>21$ units per week. When omitting this extreme group, as a sensitivity analysis, there was no evidence of a non-linear relationship $\left(\chi^{2}=0.3\right.$, d.f. $\left.=1, P=0.566\right)$ and no evidence of association between alcohol use and PLIKS (adjusted OR per 10 units $0.97,95 \%$ CI $0.72-1.31$ ) (Table 2).

We also examined trimester-specific effects of maternal alcohol use. Alcohol intake during the first and third trimesters were correlated, although insufficiently to render collinearity a problem in an analysis with both included in the same model (Pearson's coefficient 0.54). Within such a model, first trimester alcohol use (adjusted OR per 10 units 1.41, 95\% CI 0.95-2.09) but not third trimester use (adjusted OR per 10 units $0.99,95 \%$ CI $0.63-1.55$ ) was associated with increased risk of PLIKS, although the confidence intervals overlapped substantially.

We further examined possible effects of confounding by studying the effects of maternal alcohol use 4 years post-pregnancy on risk of PLIKS in the offspring. The correlation between alcohol use during and post-pregnancy was not very strong (Pearson's coefficient 0.29). There was no evidence of any association between maternal alcohol use post-pregnancy and any suspected or definite PLIKS either before or after adjusting for alcohol use

Table 3 Crude and adjusted odds ratios (OR) and $95 \% \mathrm{Cl}$ for any suspected or definite psychosis-like symptoms (PLIKS) in relation to parental tobacco use within and outside the pregnancy period (linear trend across four smoking categories)

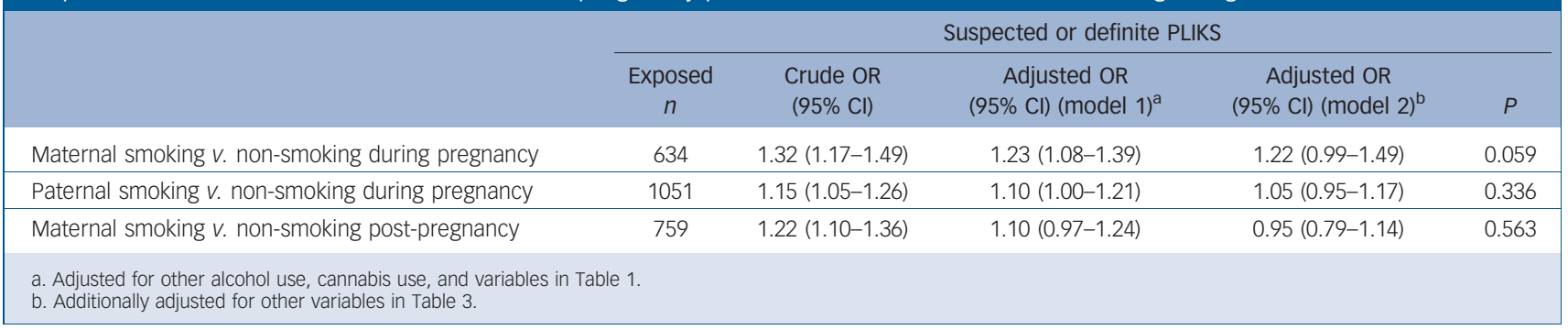


during pregnancy (LRT for both linear and quadratic terms, $\chi^{2}=4.0$, d.f. $\left.=2, P=0.139\right)$.

\section{Secondary analyses}

There were 165 children ( $2.6 \%$ of those interviewed) with definite, frequent (occurring $\geqslant$ monthly) PLIKS, and 233 (3.6\%) with suspected or definite 'bizarre' PILKS. There was no consistent pattern that associations were stronger for either of these outcomes.

\section{Missing data}

Results from the multivariable multiple-imputation models were very similar to those using the main data-set, although more precisely estimated, whether we imputed confounders only or outcome measures too.

\section{Discussion}

Maternal smoking during pregnancy was associated with an increased risk of psychotic symptoms in the children, with evidence of a dose-response effect whereby risk of PLIKS was highest in the offspring of mothers who smoked most heavily. This association was not mediated by childhood IQ or by markers of pre- or perinatal adversity. Maternal alcohol use was also associated with PLIKS, although this effect was present almost exclusively in the offspring of women drinking $>21$ units of alcohol per week in early pregnancy. Maternal cannabis use was uncommon, reducing the power to detect any association with this exposure. Although use of cannabis was also more common in offspring with PLIKS, this association was eliminated after adjusting for confounders, and particularly by maternal tobacco use.

\section{Confounding}

Maternal smoking was strongly associated with markers of adverse sociodemographic characteristics in our data, similar to previous reports, ${ }^{33}$ and similar to psychosis. $^{34}$ The sociodemographic characteristics of alcohol use in pregnancy were quite different to those of smoking, also similar to previous reports. ${ }^{35}$ The association between maternal smoking and PLIKS was attenuated by about $40 \%$ after adjusting for a broad range of confounders. However, conventional epidemiological methods for dealing with confounding may be particularly problematic when assessing intrauterine influences on offspring health outcomes. ${ }^{13}$ One approach to overcome this is to compare the effects of maternal exposure during pregnancy with those of paternal exposure, as the latter is likely to reflect, to some extent, similar confounding characteristics to the former. ${ }^{13}$ Maternal exposure during pregnancy can also be compared with maternal exposure postpregnancy, although such a comparison is more problematic, especially for smoking, given that women who continue to smoke throughout pregnancy may differ substantially from those who abstain during this period.

Estimates of association for paternal smoking and maternal smoking post-pregnancy were smaller than for smoking during pregnancy, and both were substantially attenuated after adjusting for maternal smoking during pregnancy. This lends some support for a biological effect of tobacco exposure in utero on risk of psychotic symptoms, although as the confidence intervals for paternal smoking overlap slightly with those for maternal smoking during pregnancy we cannot conclude that these exposures are clearly different from each other.

For alcohol use, adjustment for confounding had minimal effect on results, and there was no association between alcohol use in the post-pregnancy period and PLIKS independent from effects of use during pregnancy. However, the results of the sensitivity analyses indicate there was minimal association between alcohol use and PLIKS once women drinking $>21$ units of alcohol per week were excluded from the analysis. This level of drinking occurred in less than $1 \%$ of women in this cohort. Such women are likely to be very different from other women in the cohort in relation, for example, to personality traits and mental health characteristics. Despite the minimal effect of adjusting for confounders, it is difficult to be confident that this pattern of association is not because of confounding.

\section{Bias}

Although this is a large cohort, with a wealth of detailed information, missing data due to attrition and wave non-response in this cohort was not insubstantial, a problem common to other large-scale longitudinal studies. ${ }^{36,37}$ All estimates, however, were similar in the multiple-imputation analyses, indicating that attrition was unlikely to have substantially biased the results.

Some underreporting of substance use is likely to have occurred, and perhaps especially for cannabis use as this is illegal in the UK. Furthermore, a measure of weekly consumption of alcohol may not adequately capture peak levels of fetal exposure due to binge drinking patterns. Such misclassification tends to lead to underestimation of effects, although only if this were non-differential with respect to outcome.

\section{Possible biological mechanisms}

Animal studies indicate that fetal nicotine exposure can result in long-term structural and functional changes, ${ }^{7}$ including decreased neuronal density and size in the hippocampus and cortex, altered regulation of neuronal apoptosis, ${ }^{7,15}$ and increased expression of receptors for acetylcholine, which plays a critical role in brain maturation through modulation of axonogenesis and synaptogenesis. ${ }^{15}$ However, difficulties exist, both conceptually and pragmatically, in the interpretation of results from animal models in relation to effects in humans.

We are not aware of animal studies to date that have examined the effects of nicotine exposure in utero on putative endophenotypes of schizophrenia. Although endophenotypes of schizophrenia that can be modelled in animals are yet to be clearly determined this could potentially become an informative area for future research.

We observed suggestive evidence that maternal smoking during the third trimester was most strongly associated with risk of PLIKS, although results from subgroup comparisons should be interpreted cautiously. This is rather inconsistent with results from studies of famine $e^{38,39}$ and influenza, ${ }^{40,41}$ where early pregnancy exposure is associated with greatest risk of schizophrenia, but may reflect different sensitive periods of risks in brain development for different types of exposure. Maternal smoking, ${ }^{5,42}$ particularly during late pregnancy, ${ }^{43}$ is thought to lead to lower birth weight. ${ }^{7}$ However, adjusting for birth weight, as well as for gestation and 5-minute Apgar score had no effect on the results, and although these measures are likely to be rather crude markers of pre- and perinatal adversity, it seems unlikely that such adversity mediates or confounds the relationship between maternal smoking and offspring psychotic experiences.

Alcohol use is associated with adverse effects on placental development and function, ${ }^{44}$ and adverse perinatal outcomes, although evidence of adverse effects is not strong for low to moderate levels of alcohol consumption during pregnancy. ${ }^{45}$ Adverse long-term neurobehavioural and cognitive consequences of alcohol use have also been described, albeit more inconsistently than for tobacco use, in both human and animal studies. ${ }^{4}$ 
It is quite likely that biological effects of any exposures in utero will impact indirectly upon risk of psychotic phenomena, for example through effects on impulsivity, attention or subtle effects on cognition (that may not be picked up through global cognitive measures such as IQ), and which can potentially also affect development of other, non-psychotic psychopathology. In the EDSP study, maternal smoking was not associated with psychotic symptoms, or with any diagnosis of psychiatric disorder. ${ }^{20}$ Future studies that aim to increase knowledge about such pathways will help our understanding of the mechanisms underlying disease pathogenesis.

Although at present the status of non-clinical psychosis-like experiences in relation to rare clinical disorders such as schizophrenia is not clear, results from two cohort studies ${ }^{46,47}$ indicate that the presence of such symptoms may lead to increased risk of clinically important psychotic disorders in later life. Furthermore, as such symptoms are not uncommon in population-based studies ${ }^{46,48-51}$ and are associated with decreased occupational and social functioning over time, ${ }^{47,52}$ these phenomena may have a large impact on population health and quality of life outside the arena of clinical services, in the same way as depression does. This further highlights the importance of understanding the mechanisms underlying the aetiology of non-clinical PLIKS.

In our cohort, approximately $19 \%$ of adolescents attending the PLIKSi had mothers who smoked during pregnancy. If our results for the association between maternal smoking and PLIKS are nonbiased and truly reflect a causal relationship, we can estimate that about $20 \%$ of adolescents in this cohort would not have developed psychotic symptoms if their mothers had not smoked (the population attributable fraction). Therefore, although the effect size of association for maternal smoking is rather modest, the frequency of this exposure means that maternal smoking may nevertheless be an important risk factor for the development of psychotic experiences in the population.

\section{Implications}

Observational studies are limited in determining causality due to potential problems of residual confounding. We observed an association between maternal, but not paternal, smoking during pregnancy and risk of psychotic symptoms in the offspring, consistent with accumulating evidence from animal models of adverse effects on brain development from in utero nicotine exposure. These findings suggest that risk factors for development of non-clinical psychotic experiences may operate during early development. Future studies of how in utero exposure to tobacco affects cerebral development and function may lead to increased understanding of the pathogenesis of psychotic phenomena.

\footnotetext{
Stanley Zammit, PhD, Department of Psychological Medicine, School of Medicine, Cardiff University, and University of Bristol, UK; Kate Thomas, BSC MSC, Department of Social Medicine, University of Bristol; Andrew Thompson, MA, MMedSci, MRCPsych, Academic Unit of Psychiatry, University of Bristol; Jeremy Horwood, BSC, Department of Social Medicine, University of Bristol; Paulo Menezes, PhD, Academic Unit of Psychiatry, University of Bristol; David Gunnell, PhD, Department Acade of Social Medicine, University of Bristol, Chris Hollis, PhD DCH MCPsych, Division of Psychiatry, University of Nottingham, UK; Dieter Wolke, Dip (Psych) PhD, Departmen of Psychology and Health Research Institute, University of Warwick, UK; Glyn Lewis PhD, Glynn Harrison, MD FRCPsych, Academic Unit of Psychiatry, University of Bristol UK

Correspondence: Stanley Zammit, Department of Psychological Medicine, School of Medicine, Cardiff University, Heath Park, Cardiff CF14 4XN, Wales, UK. Email: zammits@Cardiff.ac.uk

First received 2 Dec 2008, accepted 9 Mar 2009
}

\section{Funding}

This study was funded by the Wellcome Trust grant No. GR072043MA. S.Z. is funded through a Clinician Scientist Award funded by the National Assembly for Wales. The UK Medical Research Council, the Wellcome Trust and the University of Bristol provide core support for ALSPAC.

\section{Acknowledgements}

We are extremely grateful to all the families who took part in this study, the midwives for their help in recruiting them, and the whole ALSPAC team, which includes interviewers, computer and laboratory technicians, clerical workers, research scientists, volunteers, managers, receptionists and nurses.

\section{References}

1 Office for National Statistics. Statistics on Smoking: England 2006. ONS, 2006.

2 NHS Information Centre. Statistics on Alcohol: England 2007. The Information Centre, 2007.

3 Fried PA. Conceptual issues in behavioral teratology and their application in determining long-term sequelae of prenatal marihuana exposure. J Child Psychol Psychiatry 2002; 43: 81-102.

4. Huizink AC, Mulder EJ. Maternal smoking, drinking or cannabis use during pregnancy and neurobehavioral and cognitive functioning in human offspring. Neurosci Biobehav Rev 2006; 30: 24-41.

5 Andres RL, Day MC. Perinatal complications associated with maternal tobacco use. Semin Neonatol 2000; 5: 231-41.

6 Ernst M, Moolchan ET, Robinson ML. Behavioral and neural consequences of prenatal exposure to nicotine. J Am Acad Child Adolesc Psychiatry 2001; 40: 630-41.

7 Winzer-Serhan UH. Long-term consequences of maternal smoking and developmental chronic nicotine exposure. Front Biosci 2008; 13: 636-49.

8 Fried PA, Watkinson B, Gray R. Differential effects on cognitive functioning in 13- to 16-year-olds prenatally exposed to cigarettes and marihuana. Neurotoxicol Teratol 2003; 25: 427-36.

9 Mortensen EL, Michaelsen KF, Sanders SA, Reinisch JM. A dose-response relationship between maternal smoking during late pregnancy and adult intelligence in male offspring. Paediatr Perinat Epidemiol 2005; 19: 4-11.

10 Fergusson DM, Woodward LJ, Horwood LJ. Maternal smoking during pregnancy and psychiatric adjustment in late adolescence. Arch Gen Psychiatry 1998; 55: 721-7.

11 Linnet KM, Dalsgaard S, Obel C, Wisborg K, Henriksen TB, Rodriguez A, et al. Maternal lifestyle factors in pregnancy risk of attention deficit hyperactivity disorder and associated behaviors: review of the current evidence. Am J Psychiatry 2003; 160: 1028-40.

12 Thapar A, Fowler T, Rice F, Scourfield J, van den Bree $M$, Thomas $H$, et al. Maternal smoking during pregnancy and attention deficit hyperactivity disorder symptoms in offspring. Am J Psychiatry 2003; 160: 1985-9.

13 Smith GD. Assessing intrauterine influences on offspring health outcomes: can epidemiological studies yield robust findings? Basic Clin Pharmacol Toxicol 2008; 102: 245-56.

14 Dwyer JB, Broide RS, Leslie FM. Nicotine and brain development. Birth Defects Res C Embryo Today 2008; 84: 30-44.

15 Slotkin TA. Cholinergic systems in brain development and disruption by neurotoxicants: nicotine, environmental tobacco smoke, organophosphates. Toxicol Appl Pharmacol 2004; 198: 132-51.

16 Sundram S. Cannabis and neurodevelopment: implications for psychiatric disorders. Hum Psychopharmacol 2006; 21: 245-54.

17 Cannon M, Jones PB, Murray RM. Obstetric complications and schizophrenia: historical and meta-analytic review. Am J Psychiatry 2002; 159: 1080-92.

18 Jones $\mathrm{PB}$, Rantakallio $\mathrm{P}$, Hartikainen $\mathrm{AL}$, Isohanni M, Sipila P. Schizophrenia as a long-term outcome of pregnancy, delivery, and perinatal complications: a 28-year follow-up of the 1966 north Finland general population birth cohort. Am J Psychiatry 1998; 155: 355-64.

19 Sacker A, Done DJ, Crow TJ, Golding J. Antecedents of schizophrenia and affective illness. Obstetric complications. Br J Psychiatry 1995; 166: 734-41.

20 Spauwen J, Krabbendam L, Lieb R, Wittchen HU, van OS J. Early maternal stress and health behaviours and offspring expression of psychosis in adolescence. Acta Psychiatr Scand 2004; 110: 356-64.

21 Horwood J, Salvi G, Thomas K, Duffy L, Gunnell D, Hollis C, et al. IQ and nonclinical psychotic symptoms in 12-year-olds: results from the ALSPAC birth cohort. Br J Psychiatry 2008; 193: 185-91. 
22 Golding J, Pembrey M, Jones R. ALSPAC - the Avon Longitudinal Study of Parents and Children. I. Study methodology. Paediatr Perinat Epidemiol 2001; 15: $74-87$

23 Shaffer $D$, Fisher $P$, Lucas CP, Dulcan MK, Schwab-Stone ME, NIMH Diagnostic Interview Schedule for Children Version IV (NIMH DISC-IV): description, differences from previous versions, and reliability of some common diagnoses. J Am Acad Child Adolesc Psychiatry 2000; 39: 28-38.

24 World Health Organization. Schedules for Clinical Assessment in Neuropsychiatry. American Psychiatric Research, 1994.

25 American Psychiatric Association. Diagnostic and Statistical Manual of Mental Disorders (4th edn) (DSM-IV). APA, 1994.

26 World Health Organization. The ICD-10 Classification of Mental and Behavioural Disorders: Clinical Descriptions and Diagnostic Guidelines. WHO 1992.

27 Office for Population Censuses and Surveys. Standard Occupational Classification 1990. OPCS, 1990

28 Cox JL, Holden JM, Sagovsky R. Detection of postnatal depression. Development of the 10-item Edinburgh Postnatal Depression Scale. $\mathrm{Br} \mathrm{J}$ Psychiatry 1987; 150: 782-6.

29 Wechsler D. Manual for theWechsler Intelligence Scale for Children (3rd edn) (WISC-III). Psychological Corporation, 1991.

30 Raghunathan TE, Lepkowski JM, Van Hoewyk J, Solenberger P. A multivariate technique for multiply imputing missing values using a sequence of regression models. Surv Methodol 2001; 27: 85-95.

31 Royston P. Multiple imputation of missing values. The Stata Journal 2004 3: $227-41$.

32 Kirkwood BR, Sterne JAC. Regression modelling. In Essential Medical Statistics (eds BR Kirkwood, JAC Sterne): 337-9. Blackwell Science, 2003.

33 Spencer N. Explaining the social gradient in smoking in pregnancy: early life course accumulation and cross-sectional clustering of social risk exposures in the 1958 British national cohort. Soc Sci Med 2006; 62: 1250-9.

34 Wicks S, Hjern A, Gunnell D, Lewis G, Dalman C. Social adversity in childhood and the risk of developing psychosis: a national cohort study. Am J Psychiatry 2005; 162: 1652-7.

35 Day NL, Cottreau CM, Richardson GA. The epidemiology of alcohol, marijuana, and cocaine use among women of childbearing age and pregnan women. Clin Obstet Gynecol 1993; 36: 232-45.

36 Callaway LK, McIntyre HD, O'Callaghan M, Williams GM, Najman JM, Lawlor DA. The association of hypertensive disorders of pregnancy with weight gain over the subsequent 21 years: findings from a prospective cohort study. Am J Epidemiol 2007; 166: 421-8.

37 Plewis I, Calderwoof L, Hawkes D, Nathan G. National Child Development Study and 1970 British Cohort Study Technical Report: Changes in the NCDS and BCS7O Populations and Samples Over Time. Centre for Longitudinal Studies, Institute of Education, 2004
38 St Clair D, Xu M, Wang P, Yu Y, Fang Y, Zhang F, et al. Rates of adult schizophrenia following prenatal exposure to the Chinese famine of 19591961. JAMA 2005: 294: 557-62.

39 Susser E, Neugebauer R, Hoek HW, Brown AS, Lin S, Labovitz D, et al. Schizophrenia after prenatal famine. Further evidence. Arch Gen Psychiatry 1996; 53: 25-31.

40 Brown AS, Begg MD, Gravenstein S, Schaefer CA, Wyatt RJ, Bresnahan M, et al. Serologic evidence of prenatal influenza in the etiology of schizophrenia. Arch Gen Psychiatry 2004; 61: 774-80.

41 Byrne M, Agerbo E, Bennedsen B, Eaton WW, Mortensen PB. Obstetric conditions and risk of first admission with schizophrenia: a Danish national register based study. Schizophr Res 2007; 97: 51-9.

42 Wang $X$, Tager IB, Van Vunakis $H$, Speizer FE, Hanrahan JP. Maternal smoking during pregnancy, urine cotinine concentrations, and birth outcomes. A prospective cohort study. Int J Epidemiol 1997; 26: 978-88.

43 Jaddoe VW, Verburg BO, de Ridder MA, Hofman A, Mackenbach JP, Moll HA, et al. Maternal smoking and fetal growth characteristics in different periods of pregnancy: the generation R study. Am J Epidemiol 2007; 165: 1207-15.

44 Burd L, Roberts $\mathrm{D}$, Olson $\mathrm{M}$, Odendaal $\mathrm{H}$. Ethanol and the placenta: a review. J Matern Fetal Neonatal Med 2007; 20: 361-75.

45 Henderson J, Gray R, Brocklehurst P. Systematic review of effects of lowmoderate prenatal alcohol exposure on pregnancy outcome. BJOG 2007: 114: $243-52$.

46 Poulton R, Caspi A, Moffitt TE, Cannon M, Murray R, Harrington H. Children's self-reported psychotic symptoms and adult schizophreniform disorder: a 15-year longitudinal study. Arch Gen Psychiatry 2000; 57: 1053-8.

47 Hanssen M, Bak M, Bijl R, Vollebergh W, van Os J. The incidence and outcome of subclinical psychotic experiences in the general population. Br J Clin Psychol 2005; 44: 181-91.

48 Eaton WW, Romanoski A, Anthony JC, Nestadt G. Screening for psychosis in the general population with a self-report interview. J Nerv Ment Dis 1991; 179: 689-93.

49 Johns LC, Cannon M, Singleton N, Murray RM, Farrell M, Brugha T, et al. Prevalence and correlates of self-reported psychotic symptoms in the British population. Br J Psychiatry 2004; 185: 298-305.

50 van Os J, Hanssen M, Bijl RV, Vollebergh W. Prevalence of psychotic disorder and community level of psychotic symptoms: an urban-rural comparison. Arch Gen Psychiatry 2001; 58: 663-8.

51 Wiles NJ, Zammit S, Bebbington P, Singleton N, Meltzer H, Lewis G. Selfreported psychotic symptoms in the general population. Results from the longitudinal study of the British National Psychiatric Morbidity Survey. $\mathrm{Br} J$ Psychiatry 2006; 188: 519-26.

52 Rossler W, Riecher-Rossler A, Angst J, Murray R, Gamma A, Eich D, et al. Psychotic experiences in the general population: a twenty-year prospective community study. Schizophr Res 2007; 92: 1-14. 